Urol. int. 1965;19:VII

\title{
Discorso introduttivo
}

Quando decidemmo di organizzare a Napoli una riunione scienti-fica passammo in rassegna quello che sarebbe potuto essere l'argo-mento della riunione. Esso doveva soddisfare le seguenti condizioni: essere di interesse generale; essere nuovo ma non tanto da poterne dire poco; essere di interesse urologico, ma non soltanto urologico. Ci sembrò che la endocrinochirurgia soddisfacesse a queste considera-zioni e ne scegliemmo tre capitoli: L'endocrinochirurgia nel cancro della prostata La paratiroidectomia nella calcolosi renale L'endocrinochirurgia negli stati intersessuali che formano ogget-to dei tre Simposii di cui adesso inizieremo i lavori.

In un periodo di estrema specializzazione della medicina alcuni capitoli della patologia e della clinica risultano come delle «terre di nessuno» che vengono pertanto trascurate da tutti. Lo studio di questi capitoli pone dei problemi multipli e differentissimi, che devono essere affrontati contemporaneamente da differenti specialisti.

In questo intendimento noi abbiamo organizzato le Giornate Internazionali di Endocrinochirurgia.

C. Ci auguriamo che da questo studio in comune possano venirfuori non conclusioni di valore universale

il che sarebbe pretenderetroppo

ma una migliore conoscenza di alcuni punti

un allargamentodelle nostre vedute in modo che queste Giornate potranno rappresen-tare non un punto di arrivo

ma una base di partenza per futuri in-contri e studi. M. Sorrentino 